\title{
Affordable Near-Term Burning-Plasma Experiments
}

\author{
Dale M. Meade and Robert D. Woolley \\ Princeton University \\ P.O. Box 451, Princeton, N. J. 08543
}

\begin{abstract}
Fusion energy is a potential energy source for the future with plentiful fuel supplies and is expected to have benign environmental impact. The issue with fusion energy has been the scientific feasibility, and recently the cost of this approach. The key technical milestone for fusion is the achievement of a self-sustained fusion fire, ignition, in the laboratory. Despite 40 years of research and the expenditure of almost \$20B worldwide, a self-sustained fusion fire has not yet been produced in the laboratory. The fusion program needs a test bed, preferably more than one, where the dynamics of a burning plasma can be studied, optimized and understood so that the engineering requirements for an engineering test reactor can be determined. Engineering and physics concepts must be developed within the next decade that will lead to an Affordable Burning Plasma Experiment if fusion is going to be perceived as making progress toward a potential long range energy source.
\end{abstract}

\section{Introduction}

The tokamak approach to fusion plasma confinement has made outstanding progress with the $\mathrm{n} \tau_{\mathrm{E}} \mathrm{T}$ parameter (fuel density x plasma energy confinement time $\mathrm{x}$ fuel ion temperature) increasing by a factor of 1000 over the past 25 years. Present experimental results from the larger tokamaks are approaching the break-even regime of $\mathrm{Q} \sim 1$ using power plant D-T fuel $[1,2]$. However, the $n \tau_{E} T$ parameter achieved so far is only $10 \%$ of that required for a self sustained burning plasma. For the past 10 years an international effort has been underway to design a tokamak that would achieve sustained ignition for pulse lengths $\sim 1000$ s long at fusion power production levels of 1,500 MWt. This device is not designed to produce net electrical power. The mission of this device is to demonstrate the scientific and technological feasibility of magnetic fusion and is to be a flexible experimental facility for the study of advanced tokamak modes under burning plasma conditions. This design concept, International Thermonuclear Experimental Reactor (ITER) is estimated to cost $\sim \$ 10 \mathrm{~B}$ with a construction period of about 10 years [3].

\section{Affordable Burning Plasma Experiment}

The general level of competing energy technologies can be understood by comparing the cost of the proposed fusion ignition experiment with existing power plants The cost of existing coal plants that produce 1,500 MWe cost about \$1.5B to construct (Laramie River Station, Fig. 1) and are expected to remain in this price range for the next several decades [4]. Fusion must succeed on its own merits by competing economically with existing power producing technologies rather than assuming the existing energy technologies will fail sometime in the future.

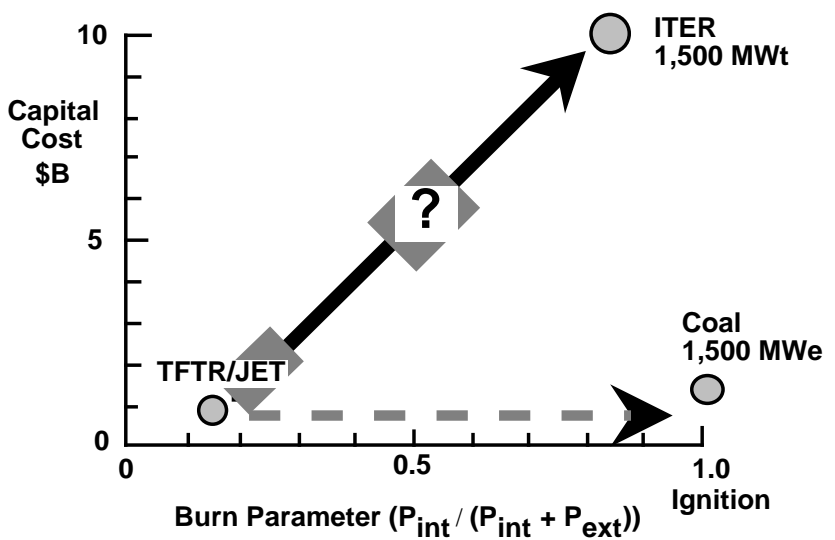

Fig. 1. Comparison of the capital cost of ITER with an operating coal power plant.

Therefore, power producing fusion systems must be sought in the few $\$ \mathrm{~B}$ range, this suggests that next step burning plasma experiments should have a construction cost less than $\sim$ \$1B. Historically, in the U. S., proposals for ignition physics experiments costing $>\$ 1 \mathrm{~B}$ have not been supported even under better funding conditions those that exist today. These considerations imply that burning plasma experiments should cost significantly less than $\$ 1 \mathrm{~B}$, preferably in the range of 0.3 to $0.5 \$ \mathrm{~B}$ if they are to be funded on a reasonable time scale.

\section{A. Stair-step Approach / Staged Focused Mission}

If these lower cost affordable devices are to be realized, the mission for a burning-plasma experiment must be focused and reduced to the minimum that will address the key burning-plasma physics issues. The reactor prototype requirement must be avoided since the reactor physics regimes or technologies are not known at this time. In addition, the mission should be staged to allow incremental deliverables and funding rather than trying to do everything at once. An example of a staged approach to full burning plasma performance is shown in Table I and is compared to the various stages of demonstrating the scientific feasibility of heavier than air flight. The present deuterium-tritium (D-T) experiments on TFTR and JET are investigating burning plasmas at Level 0 and have extended previous studies that used only deuterium plasmas [1,2]. TFTR has studied some aspects of Level 1 performance with the observation of self-heating by alpha particles [5] and the destabilization of toroidal Alfvén eigen modes by alpha particles [6]. However, modifications of existing experiments or new devices are needed to fully extend burning plasma performance into the Level 1 regime. 
Table I.

Staged Performance for Affordable Burning Plasma Device

\section{Level 0 - Weakly Burning Plasma (Wind tunnel tests)} D-T plasma and alpha particle dynamics

Level 1 - Transient Core Ignition (First self-powered lift) $\delta \mathrm{P}_{\text {fusion }} / \mathrm{P}_{\text {fusion }} \sim 1$ due to alpha heating, or net energy production from burning plasma

Level 2 - Flash Ignition (First Wright Brother's flight) $\mathrm{P}_{\alpha} \sim \mathrm{P}_{\mathrm{aux}}, \quad \sim 10 \tau_{\mathrm{E}}$

Level 3 - Controlled Ignition (Controlled extended flight) $\mathrm{P}_{\alpha} \gg \mathrm{P}_{\mathrm{aux}}, \quad>100 \tau_{\mathrm{E}}$

The physics issues associated with the achievement of a self-sustained burning plasma lend themselves very well to the stair-step staged approach as illustrated in Fig. 2. The key burning-plasma parameter is the degree of selfheating $=\mathrm{P}_{\alpha} /\left(\mathrm{P}_{\alpha}+\mathrm{P}_{\mathrm{aux}}\right)$, where $\mathrm{P}_{\alpha}$, is the self-heating of the plasma by alpha particles and $\mathrm{P}_{\mathrm{aux}}$ is the externally supplied auxiliary plasma heating power. The burn parameter increases monotonically as the gain of the fusion system, $\mathrm{Q}=\mathrm{P}_{\text {fusion }} / \mathrm{P}_{\mathrm{aux}}$, increases from weakly burning, $\mathrm{Q}<<1$ to self-sustained with $\mathrm{Q}>>1$. ITER is projected to operate in steady-state with $\mathrm{Q}$ ranging from a minimum of 4 up to 10 resulting in a burn parameter from $44 \%$ to $67 \%$ [7]. Also shown on Fig. 2 are the various plasma phenomena that arise as the burn parameter is increased.

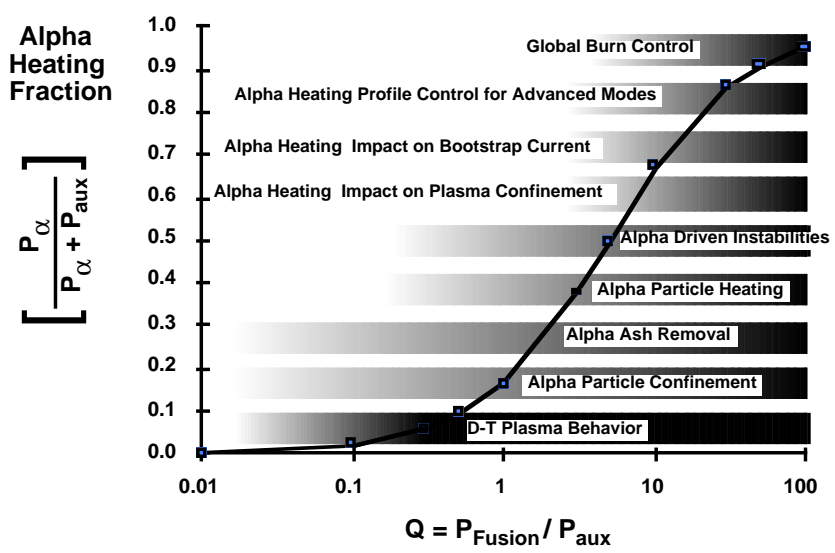

Fig. 2. Burning Plasma Physics Issues

Several of these phenomena have been studied in TFTR D-T plasmas under weak alpha heating conditions with burn parameters up to $12 \%$ in the plasma core. These studies were feasible due to the high sensitivity of the plasma diagnostic systems and the controllability of the plasma conditions that were present at the TFTR facility [1]. As the burn parameter is increased as indicated in Table I the burning plasma phenomena can be studied in greater detail as the plasma performance is optimized. The determination of plasma confinement during strong alpha heating, and the impact of the resulting pressure profile on advanced performance configurations would be an significant step for a Level I experiment.

\section{Engineering/Manufacturing Innovations are Needed.}

Magnetic fusion is faced with the same general problem as inertial fusion and accelerator builders, the unit cost of the next stage device must be reduced significantly if the device is to be affordable. An Engineering /Manufacturing Initiative should be setup to identify new approaches for design and manufacturing that will significantly reduce the cost of constructing a burning plasma facility. Some representative examples have been published and are discussed later in this paper.

Table II

Capital Costs(\$M As Spent) for TFTR Systems.

Tokamak

100

Power Supplies and Controls

Plasma Heating Systems

(including power supplies)

Diagnostics and Data Acquisition and Analysis

Buildings and Site

Total

It must be remembered that the costs for a typical fusion facility are not concentrated in the tokamak but are distributed roughly as shown in Table II, which is the approximate breakout out of capital costs for TFTR. This breakout illustrates the point that the cost of the tokamak(confinement system) is a modest part, $\sim 20 \%$, of the total facility cost. Therefore, cost saving innovations are also needed in the supporting systems such as power supplies and plasma heating as well as the tokamak. In addition, this emphasizes the importance of building at an existing site, and the need to optimize the design to maximize the utilization of available site credits.

\section{Physics Innovations are being Developed}

The standard tokamak approach does not lead to an affordable burning plasma device or to an attractive fusion reactor concept. However, several recent experimental and theoretical advances in fusion plasma physics suggest the possibility of significantly improving the confinement of fusion plasmas in tokamaks. In particular, the emerging model of plasma confinement based on marginal stability of the ion temperature gradient (ITG) modes stabilized by sheared magnetic fields and sheared plasma flows is consistent with many of the recent observations of improved confinement on TFTR, DIII-D, JT-60 and JET $[8,9]$. This model could lead to a "turbulence free tokamak" that could provide the basis for an affordable ignition device. The highest performance regimes in present day tokamaks all utilize the Hot Ion regime 
pioneered by TFTR which can be extended to the ignition regime if the ion energy confinement is neoclassical as in present experiments and the electron energy confinement is only $\sim 5$ times worse than the ion energy confinement [10]. Power handling using a radiating mantle in combination with marginal stability in the core plasma is emerging as a new approach for a practical means to maintain high performance while removing the plasma power at reasonable power densities [11]. Several new innovative physics approaches are emerging and must be tested on fusion plasmas before a tokamak engineering test reactor is constructed.

\section{Possible Technical Approaches}

A. Category I - Determine the physics and technical capability of extensions to existing $D-T$ facilities to address Level I mission elements.

The TFTR and JET facilities are the only magnetic fusion facilities in the world capable of D-T operation and each represents $\sim \$ 1 \mathrm{~B}$ investment that could serve as a base for addressing burning plasma issues at moderate burn parameters. Each facility has advantages and disadvantages that should be assessed quantitatively from the point of view of achieving results relevant to the Level I burning plasma goal with modest capital cost outlays of $<\$ 50 \mathrm{M}$. For example, TFTR has the capability of attaining significant fusion burn at the Level I mission of $\delta \mathrm{P}_{\text {fusion }} / \mathrm{P}_{\text {fusion }} \sim 1$ if the potential of the Enhanced Reversed Shear (ERS) mode could be realized as described in the numerical simulations illustrated in Fig. 3 [12]. This requires the pressure and desired magnetic profiles to be controlled to prescribed values for about $2 \mathrm{~s}$. Lower Hybrid waves for current drive and Ion Bernstein waves for pressure profile control are potential techniques for producing and controlling the ERS regime in TFTR. The achievement of significant results with D-T burning plasmas in advanced regimes would be an important step toward the development of a concept for an affordable Level III burning plasma experiment.

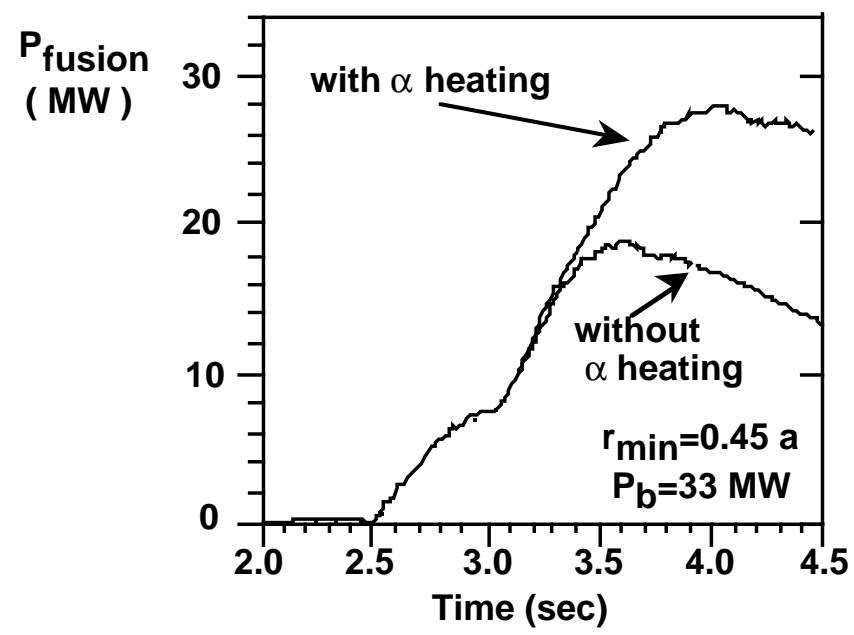

Fig. 3. Projected Performance of TFTR for Level I burning plasma goal.
Similar possibilities should be analyzed for JET and might allow the potential for net energy production from a burning plasma pulse.

B. Category II - Evaluate the physics and engineering innovations that could lead to a new core device (load assembly) that would address the Level II and III mission elements within the $\sim \$ 0.5 \mathrm{~B}$ cost goal.

\section{B1. Advanced High Field Cryogenic Copper Tokamaks}

There have been several studies of high field liquid nitrogen (LN) cooled copper magnet designs over the past two decades beginning with the Ignitor proposed by B. Coppi [13], the Compact Ignition Tokamak (CIT) [14] and the Burning Plasma Experiment (BPX) [15]. The CIT and BPX designs evolved from high risk physics to more conservative physics, and in the process the cost rose from $\sim 0.3 \mathrm{~B}$ to $\$ 1.4 \mathrm{~B}$ at which point the BPX project was canceled in 1991.

Recent experimental results have increased the confidence in achieving higher performance advanced tokamak modes and the potential of these designs should be revisited. For example, the BPX-AT study [16] which incorporated advanced tokamak modes with $\mathrm{H}=3.5, \beta_{\mathrm{N}}=$ 3.5 into the BPX design, allowed the major radius to be reduced from $2.6 \mathrm{~m}$ to $2.0 \mathrm{~m}$ thereby reducing the cost to \$0.64 M (FY 1992\$) while attaining $\mathrm{I}=6.25 \mathrm{MA}, \mathrm{B}=$ $10 \mathrm{~T}, \mathrm{Q}=25$ for pulse length $=45 \tau_{\mathrm{E}}$. Similar analyses have been done for advanced modes in Ignitor [17]. A variation of this approach is to use a single turn toroidal field coil as proposed for IGNITEX [18]. This resulted in a device with an estimated cost of \$150M.

Recently, the design of high field coils has been advanced by placing joints in the toroidal coil to reduce the stress in the center leg as was done in Alcator C-Mod [19]. Woolley has developed the design of a Cheap Ignition Tokamak using bow shaped coils with C-Mod-like joints and active LN cooling during a $100 \mathrm{~s}$ pulse that was well matched to the power systems at the TFTR site [20]. Subcooling the $\mathrm{LN}$ to $63^{\circ} \mathrm{K}$ is a further improvement. Replacing copper with aluminum in the outer legs may also have significant cost advantages

\section{B2. Advanced Physics Regimes}

Recent results with reversed magnetic shear configurations on TFTR, DIII-D and JT-60 suggest that the ion energy confinement can be increased to the neoclassical value [21, 22]. Taylor, et al [23] have analyzed a steadystate ignited $(Q \sim 8.7)$ case with negative central shear produced by significant bootstrap current in which the ion energy confinement was taken to be neoclassical, and the electrons had comparable conduction transport and significant synchrotron radiation. This configuration achieved Hot Ion mode ignition $\left(\mathrm{T}_{\mathrm{i}} \sim 80 \mathrm{keV}, \mathrm{T}_{\mathrm{e}} \sim 50\right.$ $\mathrm{keV})$. This specific study was aimed at steady-state with superconducting coils which probably drives the cost into the $>\$ 1 \mathrm{~B}$ range. There may be short pulse $\mathrm{Cu}$ coil versions of this physics regime that could take advantage of the higher fusion reactivity of the Hot Ion mode for ignition as described by J. F. Clarke [10]. 


\section{B3. Low Aspect Ratio / Advanced Tokamak}

Low aspect ratio tokamaks with $\mathrm{R} / \mathrm{a} \leq 1.5$ have been analyzed by M. Peng [24] and by R. Stambaugh [25] as potential neutron sources and as candidate fusion power sources. Recent experiments on START [26] at Culham Laboratory show promising initial results with $\beta \leq 30 \%$. Experiments on NSTX, which is presently under construction, will provide important data on the confinement scaling at low aspect ratio [27]. If the standard empirical scalings are followed with $\mathrm{H} \sim 4$, then plasma currents in the range of $30 \mathrm{MA}$ are required for ignition. On the other hand, if the sheared flow produced by large ion pressure gradients is sufficient to stabilize turbulence as suggested by the emerging models of turbulence, this approach may provide an attractive path to ignition.

C. Category III - Search for radically different approaches in both physics and engineering that offer high pay off at high risk.

There is a promising idea in this area put forward by G. V. Sheffield [28] that utilizes force-free toroidal field/ohmic heating coils [29]. This configuration produces $\mathrm{B}_{\mathrm{T}}=8.5 \mathrm{~T}$ at coil stress levels $\sim 153 \mathrm{MPa}$ while providing the magnetic flux of $66 \mathrm{~Wb}$ for inducing a plasma current of 13.5 MA. The poloidal fields are provided by a $0.23 \mathrm{~m}$ thick LN cooled $\mathrm{Cu}$ shell with a single poloidal cut. The $\mathrm{Cu}$ shell also reduces the overturning moment load on $\mathrm{TF}$ and ameliorates the effects of disruptions. The conducting shell time constant of $\sim 70$ s would allow burn pulses $\sim 20$ to $30 \mathrm{~s}$ long. The divertor fields could be energized by inductive coupling if required. Initial simulations using the Tokamak Simulation Code (TSC) confirm the general assumptions. Several open issues (startup, vessel shape, vertical stability, bootstrap current, shell cross-over currents, etc.) need to be studied. Empirical scaling suggest ohmic ignition with $2-2.5 \mathrm{x}$ L-mode. This configuration should be studied with new confinement models. While no detailed cost estimates have been done for this configuration, it is clear that this approach should be able to meet a $\$ 300 \mathrm{M}$ construction cost goal.

\section{SUMMARY}

Magnetic fusion needs to make visible progress with concrete understandable deliverables during the next five years. A sequence of staged burning plasma goals would allow progress and success even with restricted funding. Magnetic fusion needs a test bed(s) where the fundamental fusion physics and innovations can be studied and understood in the "real thing", a reacting fusion plasma. There are opportunities, such as those described above, that should be evaluated in the very near term.

\section{ACKNOWLEDGMENTS}

The authors wish to acknowledge stimulating discussions with many colleagues. This work was supported by DOE Contract No. DE-AC02-76-CHO-3073.

\section{REFERENCES}

1. D. M. Meade, "TFTR Retrospective," this conference

2. M. Keilhacker, "Deuterium-Tritium Experiments on JET and Their Implications for ITER," this conference

3. R. Aymar, "ITER Project - Status and Prospects," this conference

4. Laramie River Power Station, Web Address http://www.basinelectric.com/plants.htm

5. G. Taylor et al, Phys. Rev. Lett. 76, 2722, 1996

6. R. Nazikian et al, Phys. Rev. Lett. 78, 2976, 1997

7. Fusion Energy Advisory Committee review of ITER Detailed Design Report, April 1997, U. S. Department of Energy.

8. M. Kotschenreuther, et al, Sixteenth IAEA Fusion Energy Conference, Montreal 1996, paper CN-64/D1-5.

9. R.E. Waltz, et al, Sixteenth IAEA Fusion Energy Conference, Montreal 1996, paper CN-64/D1-6.

10. J. F. Clarke, Nucl. Fusion 20, 563, 1980

11. K. W. Hill et al, "Tests of Transport Theory and Reduced Impurity Influx with Highly Radiative Plasmas in TFTR," to be published Physics of Plasmas.

12. D. R. Mikkelson, et al, Phys. Plasmas 4(5), May 1997

13. B. Coppi et al, J. Fusion Energy 13, 2/3, 111, 1994

14. D. Sigmar et al, Thirteenth IAEA Conference on Plasma Physics and Controlled Thermonuclear Fusion Research 3, 455, 1991

15. R. J. Goldston et al, Fusion Tech. 21, 3, 1992

16. D. M. Meade and W. Reiersen, unpublished

17. B. Coppi et al, Sixteenth IAEA Fusion Energy Conference, Montreal 1996, paper CN-64/GP-14.

18. R. Carrera, et al, Thirteenth IAEA Conference on Plasma Physics and Controlled Thermonuclear Fusion Research 3, 567, 1991

19. J. H. Irby et al, "The Alcator C-Mod Tokamak and Recent Results," this conference

20. R. D. Woolley, "Design Study of Copper Toroidal Field Coils with Bow Shaping, Demountable Sliding Joints and Active Liquid Nitrogen Cooling," ANS Annual Meeting, Reno 1996

21. F. Levinton et al, Phys. Rev. Lett. 75, 4417, 1995

22. E. J. Strait et al, Phys. Rev. Lett. 75, 4421, 1995

23. T. S. Taylor et al, General Atomics Report GAA22117, 1995

24. Y.-K.M. Peng and D. J. Strickler, Nucl. Fusion 26, 576, 1986

25. R. D. Stambaugh et al, Sixteenth IAEA Fusion Energy Conference, Montreal 1996, paper CN-64/G1-2.

26. A. Sykes et al, "High Beta Performance of the START Spherical Tokamak," European Physical Society meeting, 1997, to be published in Plasma Physics and Controlled Fusion

27. C. Neumeyer et al, Engineering Overview of the National Spherical Torus Experiment (NSTX), this conference.

28. G.V. Sheffield, private communication

29. J. Kondoh, et al, "Tokamak with Force-Balanced Coil," this conference. 
